\title{
Multiple Autoimmune Syndrome With Alopecia Universalis and Immune Thrombocytopenic Purpura
}

Turki A. Alwasaidi ${ }^{1,2}$, Waleed Mustafa ${ }^{3}$, Haitham Osman ${ }^{1}$, Abdulqader Al-Hebshi ${ }^{4,5}$, Asma Alfetayeh Sr. 6

1. Hematology Division/Internal Medicine Department, Prince Mohammed Bin Abdulaziz Hospital - Ministry of National Guard Health Affairs, Madinah, SAU 2. Internal Medicine, Taibah University, Madinah, SAU 3. Hematology, University Hospital Limerick, Limerick, IRL 4. Pediatrics-Hematology Oncology, Prince Mohammed Bin Abdulaziz Hospital, Madinah, SAU 5. Pediatrics, King Saud Bin Abdulaziz University for Health Sciences, Riyadh, SAU 6. Hematology, Prince Mohammed Bin Abdulaziz Hospital, Madinah, SAU

Corresponding author: Haitham Osman, haithmm@gmail.com

\begin{abstract}
Multiple autoimmune syndrome (MAS) is a condition characterized by three or more autoimmune disorders in the same individual. The development of MAS involves genetic, immunological, and infectious factors. Here we report a case of a 40-year-old man who presented with four autoimmune diseases, namely autoimmune hypothyroidism, alopecia universalis, celiac disease, and immune thrombocytopenic purpura (ITP), which leads to a diagnosis of MAS. However, the patient does not fit in any category of MAS classification. In addition to the need for continued surveillance for the development of new autoimmune disease in predisposed patients, this case report suggests an additional fourth category of the classification of MAS that includes autoimmune hypothyroidism, alopecia universalis, celiac disease, and ITP.
\end{abstract}

Review began 01/22/2021 Review ended 01/28/2021 Published 01/31/2021

\section{(๑) Copyright 2021}

Alwasaidi et al. This is an open access article distributed under the terms of the Creative Commons Attribution License CC-BY 4.0., which permits unrestricted use, distribution, and reproduction in any medium, provided the original author and source are credited.
Categories: Internal Medicine, Allergy/Immunology, Hematology

Keywords: alopecia universalis, multiple autoimmune syndrome ( mas ), immune thrombocytopenia purpura, itp, autoimmune hypothyroidism

\section{Introduction}

Autoimmune diseases are initiated by the loss of immunological tolerance to self-antigens and that makes up, a heterogeneous group of disorders in which multiple alterations in the immune system result in targeting specific organs by immune responses or affect the body systematically [1]. Multiple autoimmune syndrome (MAS) is defined as the occurrence of at least three autoimmune diseases in the same patient [2]. It is further classified into type 1 MAS, type 2 MAS, and type 3 MAS. Celiac disease affects $1 \%$ of the general population and is an important autoimmune disease, but it is not included in the classification of MAS. However, dermatitis herpetiformis, which is one of the associated conditions with celiac disease, is one of the conditions that define type 3 MAS. Celiac disease is strongly associated with autoimmune hypothyroidism and Sjögren's syndrome in $2 \%-7 \%$ and $4.5 \%-15 \%$, respectively $[3,4]$ and is very less commonly associated with systemic lupus erythematosus (SLE). It is noteworthy that the existence of one autoimmune disease can contribute to the detection and diagnosis of other autoimmune conditions.

\section{Case Presentation}

The 40-year-old male patient is known to have hypothyroidism, which was diagnosed two years ago, and currently on thyroxin replacement therapy. The patient was diagnosed with alopecia universalis 12 months ago, when he gradually lost his all body hair over four months. He followed up by a dermatologist who treated him with local steroid injection to maintain his eyebrows hair and mustache. He was also diagnosed with iron deficiency anemia, ferritin was $20.4 \mathrm{ng} / \mathrm{ml}$ (normal: $20-360 \mathrm{ng} / \mathrm{ml}$ ), and with B12 deficiency which was $174 \mathrm{pg} / \mathrm{ml}$ (n: $187-883 \mathrm{pg} / \mathrm{ml}$ ) six months before his presentation and treated with parenteral iron (iron 200mg X 5 doses) and cyanocobalamin (vitamin B12) supplement (B12 1000 mcg X 12 injections).

He presented to the hospital after referral for incidental thrombocytopenia. His platelet count was 40×109/1 (discovered three weeks before the presentation) and dropped to $24 \times 109 / 1$ in one week. There was no history of bleeding from any site, no petechiae or easy bruising, no preceding febrile illness or flu-like symptoms no joint pain or swellings. No family history of thrombocytopenia or rheumatological disease or use of quinine or diuretics. Social history showed that he was a smoker (11 pack-year history) and he is a non-alcohol consumer.

Physical examinations showed total loss of body hair including the scalp, eyebrows, and mustache. His skin was generally erythematous; no typical malar rash or petechial rash or ecchymosis, no lymphadenopathy, or gingival hyperplasia, no hepatsplenomegaly. 


\section{Cureus}

Work up carried out included autoimmune serology which was positive for anti-thyroid peroxidase (TPO), anti-gastric parietal cell, anti-tissue transglutaminase (tTG), and anti-gliadin antibodies. Hepatitis serology, HIV, Helicobacter pylori (H. pylori) stool antigen, and anti-intrinsic factor antibodies were negative. Blood smear showed thrombocytopenia and mild leukocytosis presented as a mild left shift of neutrophilia and toxic granulations.

Because of the combination of iron deficiency and B12 deficiency, the patient underwent gastroscopy with duodenal biopsy which showed no histologic features suggestive of celiac disease or gastritis. As a part of the ITP workup, he underwent bone marrow aspiration that showed normal trilineage and adequate megakaryocytes without infiltration. Cytogenetic analysis showed normal karyotype which indicated peripheral platelet destruction in keeping with the diagnosis of ITP. An abdominal ultrasound revealed no features of chronic liver disease, no hepatomegaly, no splenomegaly or splenic atrophy, no adenopathy. His recent B12 and iron profile were in the normal range as already he received B12 and iron supplements prior to presentation to our hospital.

His thrombocytopenia was initially treated with high dose prednisolone $(1 \mathrm{mg} / \mathrm{kg})$. There was a suboptimal response in his platelet count but his alopecia universalis improved partially. Upon tapering the prednisolone dose, his hair fall recures back. After six weeks on steroid, his ITP was labeled as steroid

refractory so he went for the second-line treatment which was rituximab $375 \mathrm{mg} / \mathrm{m}^{2}$ weekly times X4 with no response in both his platelet count and alopecia universalis. Then he was switched to the 3rd line eltrombopag (thrombopoietin receptor agonist) $75 \mathrm{mg}$ orally once daily along with azathioprine 50 $\mathrm{mg}$ oral daily after which his platelet count increased and reached $106 \times 10^{9}$ for the first time and his facial and scalp hair showed good improvement; he is on current treatment almost for 12 months now. He was continued on parenteral B12 monthly and he was advised to avoid gluten-containing diet to prevent subtotal villous atrophy in the future. Table 1 shows the workup investigations.

\begin{tabular}{|c|c|}
\hline Platelet & $106(n: 150-450 \times 109 / /)$ \\
\hline Serum ferritin & 20.4 (n: $20-300$ ng/ml) \\
\hline Vitamin B12 & $210(\mathrm{n}: 187-883 \mathrm{pg} / \mathrm{ml})$ \\
\hline Serum iron & 53 (n: 49-181 mcg/l) \\
\hline Folate & 29.1 (n: 7.9-46.4 nmol/L) \\
\hline TSH & 0.90 (n: 0.35-4.94 mlU/L \\
\hline Free T4 & 16.00 (n: 9.00-19.00 pmol/L) \\
\hline TPO & 18.53 (n 1.00-16.00 IU/mL) \\
\hline Serum calcium & 9.7 (n: 8.4-10.2 mg/dl) \\
\hline ALT & $23(\mathrm{n}: 21-72 \mathrm{u} / \mathrm{l})$ \\
\hline AST & $15(n: 21-72 u / l)$ \\
\hline Intrinsic factor antibodies & $<2$ (n: up to $20 \mathrm{AU} / \mathrm{ml}$ ) \\
\hline Anti-tissue transglutaminase $\lg \mathrm{A}(\mathrm{tTG})$ antibodies & 30.93 (n: < $25 \mathrm{lU} / \mathrm{ml})$ \\
\hline Anti-tissue transglutaminase $\lg G(t T G)$ antibodies & $7.98(\mathrm{n}:<25 \mathrm{lU} / \mathrm{ml})$ \\
\hline GPA antibodies & 103.67 ( $<20$ negative $-20.1-24.9$ equivocal - >25 positive) \\
\hline Anti-endomysial IgA antibodies & Titer <1:10 (<1:10 negative - >1:10 positive) \\
\hline Anti-gliadin IgA antibodies & 27.34 (<20 is negative - >20 is positive) \\
\hline i-gliadin IgG antibodies & $5.28(<20$ is $\Rightarrow$ \\
\hline
\end{tabular}

\section{TABLE 1: Laboratory investigations.}

ALT: alanine aminotransferase; AST: aspartate aminotransferase; TSH: thyroid-stimulating hormone; T4: thyroxine; TPO: thyroid peroxidase antibodies; GPA: gastric parietal cell antibody.

\section{Discussion}




\section{Cureus}

MAS describes the presence of three or more autoimmune disease in one patient. Additionally, it is classified into three types: type 1 , type 2 , and type 3 (Table 2). This classification may help in uncovering other autoimmune diseases in the same patient [5]. The pathogenesis of MAS is not clearly understood, but many environmental factors in genetically predisposed people are thought to cause disorders of immune responses.

\begin{tabular}{|c|c|c|}
\hline Type 1 MAS & Type 2 MAS & Type 3 MAS \\
\hline Myasthenia gravis & Sjogren's syndrome & Sjogren's syndrome \\
\hline Thymoma & SLE & Myasthenia gravis and or thymoma \\
\hline Polymyositis & Rheumatoid arthritis & Addison's disease \\
\hline \multirow[t]{4}{*}{ Giant cell myocarditis } & Primary biliary cirrhosis & Type 1 diabetes mellitus \\
\hline & Scleroderma & Autoimmune haemolytic anaemia, pernicious anaemia, ITP \\
\hline & Autoimmune hypothyroidism & Autoimmune hypothyroidism \\
\hline & & Dermatitis herpetiformis \\
\hline
\end{tabular}

TABLE 2: Classification of multiple autoimmune syndrome.

MAS: multiple autoimmune syndrome; ITP: immune thrombocytopenic purpura.

The patient was diagnosed previously with autoimmune hypothyroidism based on positive serology for thyroid peroxidase (TPO) IgG antibodies. These antibodies are considered to be secondary to the thyroid damage inflicted by T cells [6]. As an advanced form of alopecia areata (loss of scalp hair), the diagnosis of AU was clinically made. Alopecia areata is associated with a variety of autoimmune diseases. The strongest association has been with autoimmune thyroid disorders which occurs in $8 \%$ to $28 \%$ of patients $[7,8]$. Patients with MAS often have a dermatological condition, usually vitiligo or alopecia areata [9]. Considering the patient's medical history of iron deficiency anemia and B12 deficiency, a serologic evaluation, to detect tissue transglutaminase and gliadin antibodies, was initiated for the diagnosis of celiac disease. Serology was positive for IgA anti-tTG and IgA anti-gliadin antibodies. Most studies describe anti-tTG antibodies as highly sensitive and specific for the diagnosis of celiac disease [10-12]. Upper endoscopy with small bowel biopsy was performed, looking for villous atrophy, but it was negative (Figure 1). Since celiac disease enteropathy can be patchy and missed due to sampling error, numerous biopsies obtained from multiple sites in the mid and distal duodenum are required to detect villous atrophy. One study suggested that a minimum of three biopsies is required to ensure that villous atrophy is detected [13]. Additionally, latent celiac disease can present with normal mucosa [14].

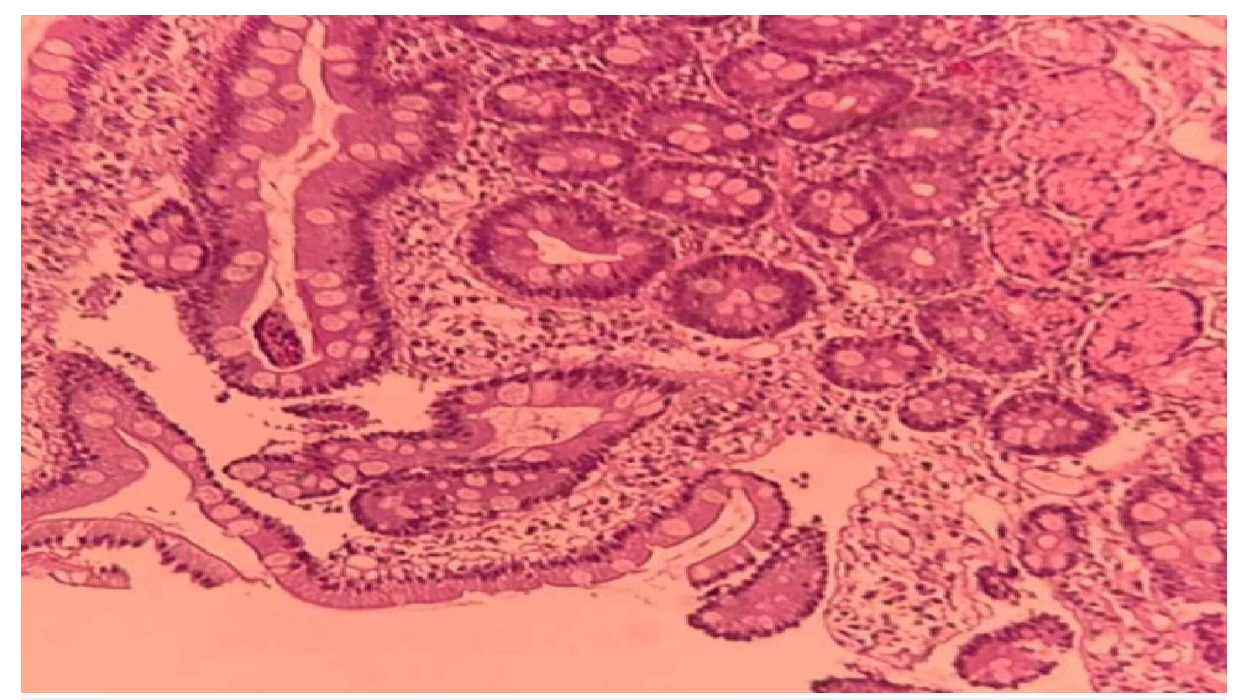

FIGURE 1: Endoscopic small bowel biopsy of the patient shows normal duodenal histology. 


\section{Cureus}

Dermatitis herpetiformis, which is frequently associated with celiac disease, is one of the conditions that classifies MAS into type 3. Although our patient did not develop it, there are associations between dermatitis herpetiformis and autoimmune thyroid disease, pernicious anemia as well as alopecia areata [15]. Furthermore, the gastric biopsy showed mild non-specific inflammation with intestinal (goblet cell type) metaplasia (Figure 2). Autoimmune gastritis, which is distinct from type B gastritis associated with H. pylori, has been linked with the development of gastric intestinal metaplasia [16]. Mild-moderate gastric dysplasia is associated with annual incidences of gastric cancer of $0.6 \%$ [17]. Serology for pernicious anemia was conducted and parietal cell antibodies were positive but the intrinsic factor antibodies, which are more specific [18,19], were negative, and hence we could not confirm the diagnosis of pernicious anemia. There is no "gold standard" test that can establish the diagnosis of ITP. The diagnosis depends on the exclusion of other causes of thrombocytopenia. Drug-induced thrombocytopenia, infections such as H. pylori, hypersplenism, liver diseases, myelodysplastic syndromes, thrombotic thrombocytopenic purpura, and congenital thrombocytopenias were all excluded. The presence of the above mention diseases, in one patient, does not fit in any of the three categories of MAS and here we suggest a fourth category that includes autoimmune hypothyroidism, celiac disease, alopecia areata, and ITP (Table 2).

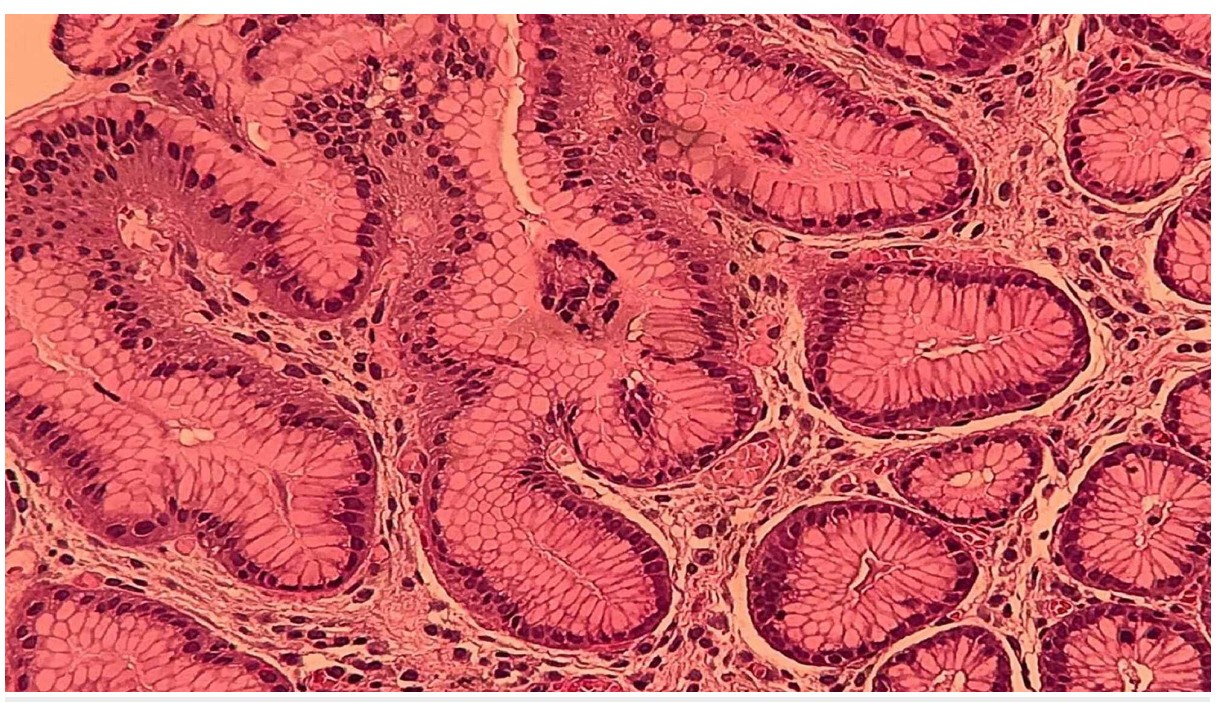

FIGURE 2: Endoscopic gastric biopsy shows mild non-specific inflammation with intestinal metaplasia. Investigations for $\mathrm{H}$. pylori were negative.

\section{Conclusions}

Our patient has MAS but does not fit in any category of MAS classification. In addition to the need for continued surveillance for the development of new autoimmune disease in predisposed patients. This case report may suggest an additional fourth category of the classification of MAS that includes autoimmune hypothyroidism, alopecia universalis, celiac disease, and ITP.

\section{Additional Information}

\section{Disclosures}

Human subjects: Consent was obtained or waived by all participants in this study. Conflicts of interest: In compliance with the ICMJE uniform disclosure form, all authors declare the following: Payment/services info: All authors have declared that no financial support was received from any organization for the submitted work. Financial relationships: All authors have declared that they have no financial relationships at present or within the previous three years with any organizations that might have an interest in the submitted work. Other relationships: All authors have declared that there are no other relationships or activities that could appear to have influenced the submitted work.

\section{References}

1. Anaya J-M: Autoimmunity and autoimmune disease: a Colombian construction beyond deconstructions . Autoimmun Rev. 2006, 5:165-166. 10.1016/j.autrev.2005.06.006

2. Anaya JM, Castiblanco J, Rojas-Villarraga A: Multiple autoimmune syndromes. Humana Press . 2008, 10.1007/978-1-60327-285-8 12

3. Spadaccino AC, Basso D, Chiarelli S, et al.: Celiac disease in North Italian patients with autoimmune thyroid diseases. Autoimmunity. 2008, 41:116-121. 10.1080/08916930701620209

4. Lidén M, Kristjánsson G, Valtýsdóttir S, et al.: Gluten sensitivity in patients with primary Sjögren's 
syndrome. Scand J Gastroenterol. 2007, 42:962-967. 10.1080/00365520701195345

5. Cojocaru M, Cojocaru IM, Silosi I: Multiple autoimmune syndrome. MAEDICA J Clin Med. 2007, 5:132-134.

6. Chiovato L, Bassi P, Santini F, et al.: Antibodies producing complement-mediated thyroid cytotoxicity in patients with atrophic or goitrous autoimmune thyroiditis. J Clin Endocrinol Metab. 1993, 77:1700-1705. 10.1210/jcem.77.6.7903315

7. Goh C, Finkel M, Christos PJ, et al.: Profile of 513 patients with alopecia areata: associations of disease subtypes with atopy, autoimmune disease and positive family history. J Eur Acad Dermatol Venereol. 2006, 20:1055-1060. 10.1111/j.1468-3083.2006.01676.x

8. Thomas EA, Kadyan RS: Alopecia areata and autoimmunity: a clinical study . Indian J Dermatol. 2008, 53:7074. 10.4103/0019-5154.41650

9. Cojocaru M, Cojocaru IM, Silosi I: Multiple autoimmune syndrome. Maedica. 2010, 5:132-134.

10. Troncone R, Maurano F, Rossi M, et al.: IgA antibodies to tissue transglutaminase: an effective diagnostic test for celiac disease. J Pediatrics. 1999, 134:166-171. 10.1016/s0022-3476(99)70410-5

11. Hopper AD, Cross SS, Hurlstone DP, et al.: Pre-endoscopy serological testing for coeliac disease: evaluation of a clinical decision tool. BMJ. 2007, 334:729. 10.1136/bmj.39133.668681.BE

12. Hopper AD, Hadjivassiliou M, Hurlstone DP, et al.: What is the role of serologic testing in celiac disease? A prospective, biopsy-confirmed study with economic analysis. Clin Gastroenterol Hepatol. 2008, 6:314-320. 10.1016/j.cgh.2007.12.008

13. Hopper AD, Cross SS, Sanders DS: Patchy villous atrophy in adult patients with suspected gluten-sensitive enteropathy: is a multiple duodenal biopsy strategy appropriate?. Endoscopy. 2008, 40:219-224. 10.1055/s2007-995361

14. Troncone R, Greco L, Mayer M, et al.: Latent and potential coeliac disease . Acta Paediatrica. 1996, 85:10-14. 10.1111/j.1651-2227.1996.tb14240.x

15. Alonso-Llamazares J, Gibson LE, Rogers RS III: Clinical, pathologic, and immunopathologic features of dermatitis herpetiformis: review of the Mayo Clinic experience. Int J Dermatol. 2007, 46:910-919. 10.1111/j.1365-4632.2007.03214.x

16. Bizzaro N, Antico A, Villalta D: Autoimmunity and gastric cancer. Int J Mol Sci. 2018, 19:377. 10.3390/ijms 19020377

17. Jencks DS, Adam JD, Borum ML, et al.: Overview of current concepts in gastric intestinal metaplasia and gastric cancer. Gastroenterol Hepatol. 2018, 14:92-101.

18. Carmel R: Reassessment of the relative prevalences of antibodies to gastric parietal cell and to intrinsic factor in patients with pernicious anaemia: influence of patient age and race. Clin Exp Immunol. 1992, 89:74-77. 10.1111/j.1365-2249.1992.tb06880.x

19. Carmel R: How I treat cobalamin (vitamin B12) deficiency. Blood. 2008, 112:2214-2221. 10.1182/blood2008-03-040253 